\title{
Determining traffic congestion utilizing a fuzzy logic model and Floating Car Data (FCD)
}

\author{
Maja Kalinic ${ }^{a, *}$, Jukka M. Krisp ${ }^{b}$ \\ ${ }^{a, b}$ Department for Applied Geoinformatics, University of Augsburg, \\ Alter Postweg 118, 86159, Augsburg, Germany \\ maja.kalinic@geo.uni-augsburg.de \\ * Corresponding author
}

\begin{abstract}
Traffic congestion is a dynamic spatial and temporal process and as such might not be possible to model with linear functions of various dependent variables. That leaves a lot of space for non-linear approximates, such as neutral networks and fuzzy logic. In this paper, the focus is on the fuzzy logic as a possible approach for dealing with the problems of measuring traffic congestion. We investigate the application of this framework on a selected case study, and use floating car data (FCD) collected in Augsburg, Germany. A fuzzy inference system is built to detect degrees of congestion on a federal highway B17. With FCD, it is possible to obtain local speed information on almost all parts of the network. This information, together with collected vehicle location, time and heading, can be further processed and transformed into valuable information in the form of trip routes, travel times, etc. Initial results are compared with traditional method of expressing levels of congestion on a road network e.g. Level of Service - LOS. The fuzzy model, with segmented mean speed and travel time parameters, performed well and showed to be promising approach to detect traffic congestions. This approach can be further improved by involving more input parameters, such as density or vehicle flow, which might reflect traffic congestion event even more realistically.
\end{abstract}

Keywords: fuzzy logic, fuzzy inference system, floating car data, traffic congestion, congestion measures

\section{Introduction}

With the rapid traffic growth, traffic networks are getting bigger, more complex and comprehensive. Accurate and reliable estimation of the traffic state is a crucial part of traffic management and control, and depends highly on how reliable and valid traffic data is (Sunderrajan et al, 2016). The acquisition of traffic data was and is performed mostly by stationary systems (e.g. inductive loop detector stations, radars, road-tube counters, etc.). More recently, Floating Car Data (FCD) has become another important traffic data source and has an increasing usage due to its lower cost and higher coverage. Its main advantage in comparison to local traffic counts is that it provides travel time along travelled paths as well as local speed information on almost all parts of the network.

Besides obtaining reliable and valid traffic data, interpreting and modelling the data is one of the crucial traffic state estimation steps. A range of methods has been suggested as measures of traffic. Pioneers in this field Turner at al. (1996), Levinson and Lomax (1996), and Boarnet at al. (1998) suggest that traffic congestion measures should demonstrate clarity and simplicity, describe the magnitude of congestion, provide a continuous range of values and include travel time. Obviously, it is hard to satisfy all the criteria with one single model, which is why it is often used combination of models to describe traffic to its best and provide useful information to road users. To overcome some of the mentioned obstacles, our idea is to approach the issue with the fuzzy logic based model. Why fuzzy logic based model? Because this model is capable of handling uncertainties and ambiguities that exist in the real physical world (Zadeh, 1965). Additionally, the model is able to address data and uncertainty regarding the accuracy of its representation of the real conditions. As real world conditions change from the roadway section to the roadway section, very often a small change in the input can significantly change the outputs.

In this paper, we document the use of floating car data (FCD) vehicle speed information and travel time needed to traverse each individual segment, as two parameters for our fuzzy inference model. Its role is to identify degrees of congestion (either no, low, mild, severe or extreme congestion) on predefined road segments of B17 federal highway. The findings are compared with traditional method of expressing levels of congestion on a road network - Level of Service (LOS).

\section{Related work}

Traffic congestion events occur when more people are trying to use a given transportation facility during a specific period than the facility itself is able to handle (Kalinic and Krisp, 2019). Congestion usually starts from a single road segment, then expands along the road and influences the nearby roads. As time passes by, those congested fragments shrink slowly, eventually reduce their coverage and finally disappear. Unfortunately, not forever 
which is why many measures for dealing with traffic and traffic related events have been developed over the course of years. Generally, these measures of traffic can be categorized into four broad groups: basic measures, ratio measures, level of service and indices.

Basic measures are related with delay estimation. Traffic delay is seen as an additional time spent into traffic in comparison to acceptable travel time (Ter Huurne and Anderson, 2014). Using traffic delay as a measure of traffic congestion requires defining threshold value as a beginning of delay. This mostly depends on the type of the road (e.g. freeways, local highways, urban roads, etc.), extent of the investigated area, urban or open space zone, etc. The biggest weakness of this method is that it does not provide information about different magnitude of congestion (e.g. low, middle, severe congestion, etc.) and there is no detailed information on travel delay on a road segment length, but rather on the whole investigated area (Aftabuzzaman, 2007). Ratio measures - delay rate, relative delay rate and delay ratio are developed by Lomax et al (1997) back in 1997. These are still in use and are based on travel rate (rate at which a road segment is travelled). However, the application of these measures is limited for a particular road type or a facility, and the calculated values cannot be used effectively for a geographic area (Hamad and Kikuchi, 2002). The Highway Capacity Manual (HBS) adopts Level of Service (LOS) approach. The LOS of a facility is determined by various traffic flow characteristics, e.g. vehicle density, volume to capacity ratio, average speed, etc. depending on the facility type (Wan et al, 2018). The scale of LOS has six discrete classes ranging from $\mathrm{A}$ (which represents completely free flow) to $\mathrm{F}$ (representing forced or breakdown flow). Despite the fact that LOS has been one of the most popular measures of traffic congestion, it has its weaknesses. This stepwise approach can sometimes be misleading, especially when the condition is near a threshold. In some cases, the condition will exceed the threshold, which might cause serious problems, especially on those sections (road segments) in which the geometric features and traffic parameters change significantly (Hamad and Kikuchi, 2002). Congestion indices are developed by the group of researchers whose idea was to include several congestion related elements in an equation to produce a single measure. These indices are mostly suitable for detecting traffic in urban areas, but are limited to a roadway segment or a particular route (He et al, 2016). In summary, congestion is an event that is caused by many factors and perceived in different ways. Hence, in the process of determining the degree of congestion, the involvement of imprecise quantities and the subjective notion of acceptability are inevitable. Additionally, it is important to take into account nature of observations and data imprecision. Moreover, to aggregate different measured quantities to yield a composite measure. We believe that fuzzy inference system can address most of these requirements.

\subsection{Fuzzy Inference System (FIS) in detecting traffic congestion}

Fuzzy logic provides an effective conceptual framework for dealing with the problems of knowledge representation in an environment of uncertainty and imprecision (Zadeh, 1998). It is effective in situations in which solutions do not need to have categorical but rather dispositional validity (Zadeh, 1973). In other words, it provides a natural way of dealing with problems in which the source of imprecision is the absence of sharply defined criteria of class membership rather than the presence of random variables (Fuller and Zimmermann, 1993). The process of determining the degree of congestion is fuzzy and has to involve imprecise quantities and subjective notion of acceptability, as well as judgement in the calculation and interpretation of the results (Mendel, 1995). Any measure related with traffic is associated with uncertainty regarding the accuracy of its representation of the real conditions. Therefore, the model needs to be capable of accomodating changes related with the roadway segments, traffic participant's experience and familiarity with the área. Additionally, it should be fairly managable in computation complexity and execution time. Building one such model, further called a fuzzy inference model for traffic congestion detection involves several phases - defining and fuzzyfying input parameters, applying fuzzy rules and operators, applying implication and aggregation method and defuzzification (Mamdani, 1975). To define input parameters is always a challenging task, which not only relays on knowledge and experience in specific field, but also data availability and reliability. Input parameters are usually crisp numerical values which undergo fuzzification by assigning them membership degrees within the fuzzy set. The simplest, yet most commonly used, membership function is the triangular function (Kosko and Mitaim, 1996). In fuzzy inference process, it is necessary to establish a mechanism, which indicates how to project input variables onto output space. This is done by specifying if - then fuzzy rules. A single fuzzy if - then rule follows the form - If $x$ is A, Then $y$ is B. In general, interpreting an ,if - then" rule involves evaluating the if-part and applying that result to the then-part. Since the output of each if - then rule is a fuzzy set, these separate sets are further aggregated into a single fuzzy set (Sugeno, 1985). Sometimes, generated fuzzy sets for each output variable might need defuzzification. In other words, converting the fuzzy output to a crisp value by using the same membership function used in the fuzzification step.

\subsection{Floating Car Data (FCD) for measuring traffic congestion}

While it is possible and worthwhile to collect data on heavily used intercity corridors via consecutively installed multiple sensors/sources, it is not economically possible to furnish all major roads in a city with sensors, nor computationally simple to rapidly analyse data in urban networks (Zhao et al., 2009). Therefore, it is necessary to concentrate on using FCD for detecting urban patterns as a single source. The principle of FCD is to collect real time traffic data, by locating the vehicle via mobile phones or 
GPS over the entire road network. Data such as car location, speed and direction of travel are sent anonymously to a central processing centre. This information is processed further, in order to derive travel time or average speeds through road segments. $\mathrm{Xu}$ et al. (2013) discuss the main issues when dealing with large data sets for obtaining meaningful traffic and congestion patterns. They suggest data cube management for storing and processing historical FCD data. Li et al. (2012) use FCD to examine variabilities in average speeds, and attempt to determine congestion locations depending on sudden decreases in average speeds in consecutive road segments. Adu-Gyamfi et al. (2015) focus on pattern recognition and time series analysis to identify congestion trends based on FCD speed data. Some studies show that using a neural network based models is highly recommended to estimate average segment speed and based on these finding determine congestion locations of the selected segments. Fabritiis et al. (2008) propose two algorithms, respectively based on artificial neural networks and pattern matching, designed to perform shortterm (15 to 30 minutes) predictions of link travel speeds by using current and near-past link average speeds estimated by FCD system. Kong et al. (2015) develop a fuzzy comprehensive evaluation method for identifying congestion locations for every 5-minute interval of data records. As seen, most of the studies concentrate mainly on determining average speed on a road segment and based on these findings estimate segment's congestion. In our approach, we utilize measured vehicle speed and calculated travel times (based on derived speed information) as input parameters for modelling. Speed is one of the most important factors because drivers' perception of the operating speeds of different routes and, consequently, of travel times and costs strongly influences their route choice and therefore traffic state (Lobo et al., 2018). Since the output (traffic congestion information) does not need to be categorically exact nor precise, we apply fuzzy logic theory to approximately model traffic congestion.

\section{Case study - detecting traffic congestion on the federal highway B17 in the city of Augsburg}

The goal of this analysis is to identify traffic behaviour (the occurrence of a traffic congestion between $1^{\text {st }}$ and $31^{\text {st }}$ of July 2018) on the federal highway B17 in Augsburg, Germany. The investigated road length is $20 \mathrm{~km}$ long and spreads from intersection where B17 meets the Augsburger street (Königsbrunn) in the south (where outer city ring ends) until the intersection with A8 highway (north). We call this chunk of a road "pleasure drive road" since it is known to be congestion free in normal daily conditions (this can be interrupted with accidents, construction works, special events, etc.). Total road length is divided into 16 road segments based on intersection infrastructure. The segment always ends or starts at the intersection, road exit or entrance. The driving direction is south north with two lanes. Figure 1 shows the case study area with odd numbered segments coloured in grey and even numbered segments in yellow.

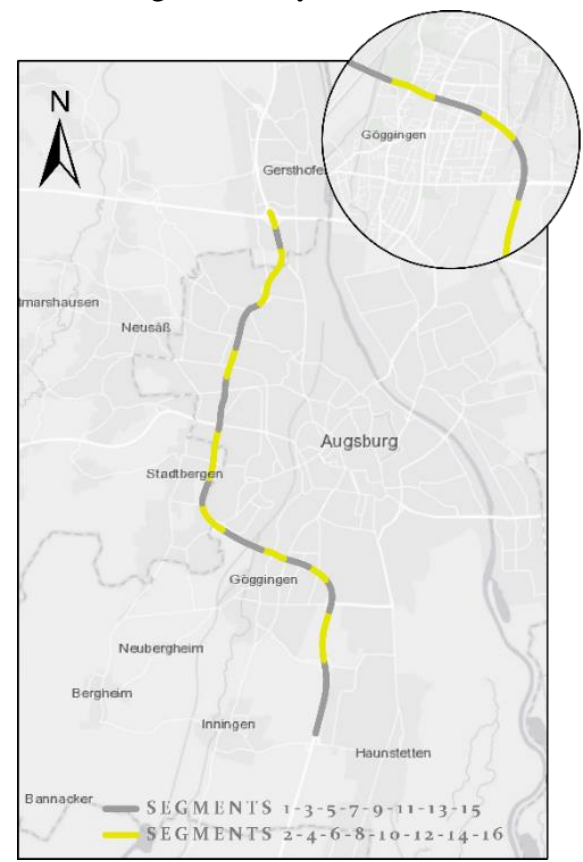

Figure 1: Chunk of federal highway B17 in Augsburg, segmented based on intersection infrastructure

\subsection{Floating Car Data (FCD) processing for detecting traffic congestion on $\mathrm{B} 17$}

We use floating car data collected in the city of Augsburg from $1^{\text {st }}-31^{\text {st }}$ of July 2018. The row data carries the information about FCD records gathered in 10s interval. Besides position (latitude and longitude), the dataset carries the information about the speed which vehicles had at the moment of recording, as well as direction of movement (heading). These two additional information allow data aggregation on hourly level, taking into account both direction and road segment length. The information about speed, available from raw data, comes in form of instantaneous speed. For the analysis, the space (segment) mean speed is better because it is statistically more stable than the time-mean speed, particularly for short roadway segments or small travel times (Wardrop, 1952). Additionally, it is associated with a specified length of roadway, whereas the time-mean speed takes into account single point along a roadway over time. Space (segment) mean-speed is calculated as a normal arithmetic average of the individual vehicle speeds in a 1-hour time interval and divided with the area of investigation (segment length). With the space-mean speed information, it is possible to calculate average travel time that the vehicles needed to transit through the specific segment. Travel time is the ratio between segment length and speed, and it is calculated for each segment on an hourly basis. Table 1 shows segment lengths, speed limits on each individual segment as well as travel time needed to traverse each segment. 


\begin{tabular}{|l|l|l|l|}
\hline & L' & SI' & TT', \\
\hline S1 & 2,4 & 120 & 72 \\
S2 & 1,6 & 120 & 48 \\
S3 & 1,1 & 70 & 54 \\
S4 & 0,7 & 70 & 36 \\
S5 & 0,8 & 70 & 42 \\
S6 & 0,8 & 70 & 42 \\
S7 & 1,4 & 70 & 72 \\
S8 & 1,1 & 70 & 54 \\
S9 & 0,9 & 70 & 54 \\
S10 & 1,6 & 60 & 96 \\
S11 & 1,8 & 60 & 108 \\
S12 & 1,0 & 50 & 72 \\
S13 & 1,8 & 60 & 108 \\
S14 & 2,1 & 100 & 78 \\
S15 & 0,6 & 100 & 24 \\
S16 & 0,5 & 100 & 18 \\
\hline
\end{tabular}

" length in kilometres $(\mathrm{km})$, " speed limit in $\mathrm{km} / \mathrm{h}$, ", travel time in seconds (s)

Table 1: Calculated segment lengths, speed limits and travel time on the selected chunk of the B17 motorway

Additionally, these two parameters (space-mean speed and travel time) are used afterwards for calculating Levels of Service (LOS) based on Highway Capacity Manual for German road network (HBS, 2015). This way, it is possible to compare detected LOC values with traditionally calculated LOS values, and discuss (fuzzy) model performance.

\subsection{Fuzzy inference system for detecting traffic congestion on B17}

We develop a fuzzy inference system (FIS) to detect congestion levels (no congestion, low, mild, severe and extreme congestion) on the predifined road segments (116). The FIS has two input variables - segment mean speed and travel time. Input variables are fuzzified with four and three linear membership functions respectively. For the speed those are normal, stable, slow, and very slow speed, and for the travel time - normal, acceptable and non acceptable travel time. In both cases, membership functions overlap with $30-50 \%$ to ensure smooth output. As an output value, we wish to have a parameter which shows how congested investigated area (segments) is. For this cause, five linguistic varibales describe the output parameter (namely no congestion, low, mild, severe and extreme congestion). We further specifiy twelve if-then fuzzy rules to map the input onto desired output. Figure 2 shows the fuzzy model for detecting degree of congestion on B17, with two input parametes - segment mean speed and travel time and one output parameter - degree of congestion.

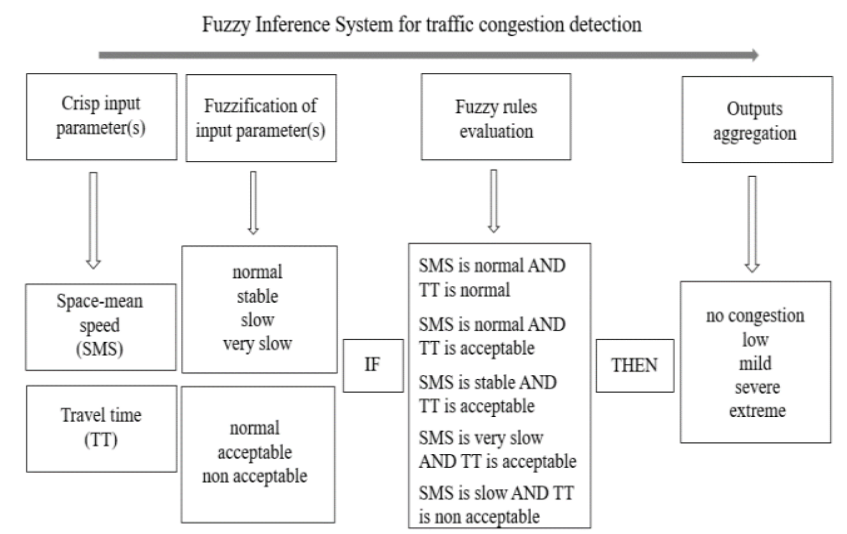

Figure 2: Fuzzy inference model for traffic congestion detection with two input parameters (segment-mean speed and travel time) and one output parameter (degree of congestion)

Predefined road segments on B17 have different speed limits and are not of the same length. In other words, vehicles have to operate with correspondence to defined speed limitations and require unique travel times to traverse each individual segment. Therefore, we take that into account and build individual model for each segment to reflect the traffic at its best. There are 16 fuzzy models in total, which are meant to refelect the traffic behavour on the selected chunk of the road.

\subsection{Calculating Level of Service (LOS) values for model evaluation}

The "classic" Level of Service (LOS) methodology is perhaps the oldest and one of the mostly used measures to describe traffic congestion. Since the fuzzy models use two parameters (space-mean speed and travel time) for describing the congestion on a chunk of B17 federal highway, the same parameters are used to estimate LOS. Based on default values proposed by German Highway capacity manual (HBS, 2015), we derive LOS values for both parameters on the investigated motorway stretch. Table 2 shows values of LOS proportional to default values (from HBS) and calculated based on the segment speed limits, and travel time needed to traverse each of the segments.

\begin{tabular}{|c|c|c|}
\hline $\begin{array}{c}\text { Level of } \\
\text { Service } \\
\text { (LOS) }\end{array}$ & $\begin{array}{l}\text { Space-mean speed of a } \\
\text { passenger vehicle } \\
\text { (in km/h)* }\end{array}$ & $\begin{array}{l}\text { Average time } \\
\text { delay } \\
\text { (in seconds) }\end{array}$ \\
\hline $\mathrm{A}$ & $>120 / 100 / 70 / 60 /$ & $<20$ \\
& 50 & $<35$ \\
$\mathrm{~B}$ & $>105 / 85 / 70 / 55 / 50$ & $<50$ \\
$\mathrm{C}$ & $>90 / 75 / 60 / 45 / 35$ \\
$\mathrm{D}$ & $>75 / 60 / 45 / 30 / 20$ & $<70$ \\
$\mathrm{E}$ & $>60 / 45 / 30 / 15 / 15$ & $>70$ \\
$\mathrm{~F}$ & $<60 / 45 / 30 / 15 / 15$ & $>90$ \\
\hline
\end{tabular}

Table 2: LOS values

*The LOS is valid for the segments where the speed limit is $120 \mathrm{~km} / \mathrm{h}$. Since not all segments on the investigated B17 road chunk have the same speed limits, other values are proportionally calculated and added to the table (e.g. for 
the speed limit of $100 \mathrm{~km} / \mathrm{h}-$ LOS B is $85 \mathrm{~km} / \mathrm{h}$, LOS C is $75 \mathrm{~km} / \mathrm{h}$, etc.). Having this classification table at hand, enables us to compare standard values of space mean speed and travel time(s) against those derived from the FCD.

\section{Results and Discussion}

Fuzzy models deliver Level of Congestion (LOC) values for all sixteen road segments, between 01-31 ${ }^{\text {st }}$ of July, 2018. For comparison with "classic" LOS values, fuzzy outputs are defuziffied. In other words, fuzzy sets that models provide (e.g. no congestion, low, mild congestion, etc.) are "translated" into crisp, numerical values using Sugeno (1985) and Lee (1990) centroid method. Table 4 shows five investigated levels of congestion (no congestion, low, mild, severe and extreme congestion) translated into numerical values, where values closer to 0 indicate congestion free flow, while values closer to 1 extreme congestion conditions.

\begin{tabular}{|l|l|}
\hline $\begin{array}{c}\text { Level of Congestion - } \\
\text { LOC }\end{array}$ & \multicolumn{1}{|c|}{$\begin{array}{c}\text { Defuzzified values } \\
(0-1)\end{array}$} \\
\hline No congestion & $<0,55$ \\
Low congestion & $<0,65$ \\
Mild congestion & $<0,75$ \\
Severe congestion & $<0,85$ \\
Extreme congestion & $<0,95$ \\
\hline
\end{tabular}

Table 3: Defuzzifed values of detected LOC's

Using the defuzzified classes from the Table 3 and LOS values from a Table 2, levels of congestion are compared. Figure 3 shows the overall traffic behavior on the federal highway B17 based on a) fuzzy models, b) level of service - space mean speed and c) level of service - travel delay. All three methods deliver slightly different results. Fuzzy models describe B17 road as mostly congestion free, with some elevated levels on the $2^{\text {nd }}, 7^{\text {th }}, 15^{\text {th }}$ and $3^{\text {rd }}$ segment, namely low to mild congestion. Level of Service (LOS) based on space mean speed describes traffic slightly worse, with $3^{\text {rd }}, 4^{\text {th }}, 5^{\text {th }}, 6^{\text {th }}, 7^{\text {th }}, 9^{\text {th }}, 10^{\text {th }}, 11^{\text {th }}, 13^{\text {th }}$ segment being shown as low and $12^{\text {th }}$ segment as mild congestion. Last, Level of Service (LOS) based on travel delay appear to be noticeably different from both (previous) measures. It shows that the investigated area is mostly under mild congestion, with $2^{\text {nd }}, 6^{\text {th }}$ and $7^{\text {th }}$ segment being even severely congested. All of the five levels of congestion are differentiated with different colours, where green means no congestion, yellow - low congestion, orange - mild congestion, dark red - severe congestion and black extreme congestion (some of the colours are not visible on the Figure 3 as corresponding levels of congestion are not detected on the selected road chunk).

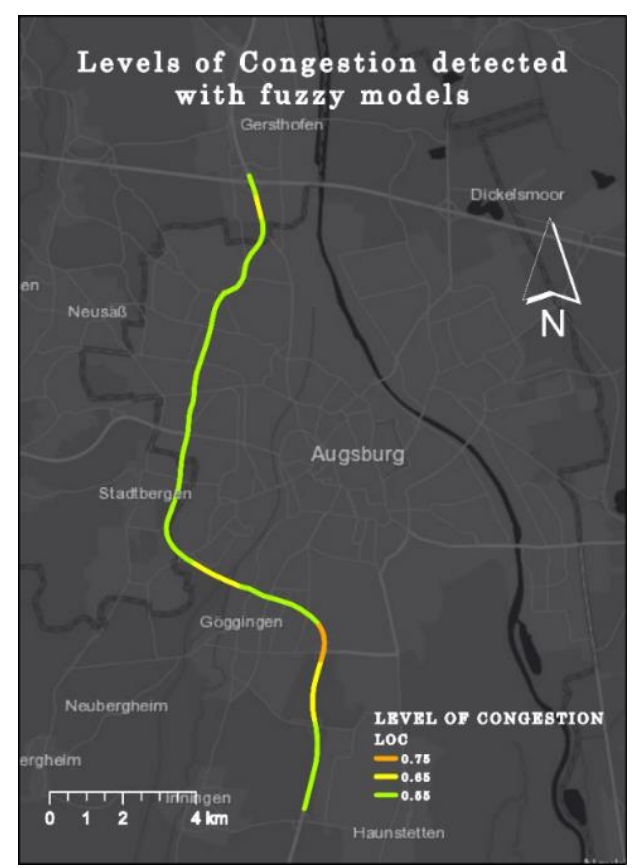

a)

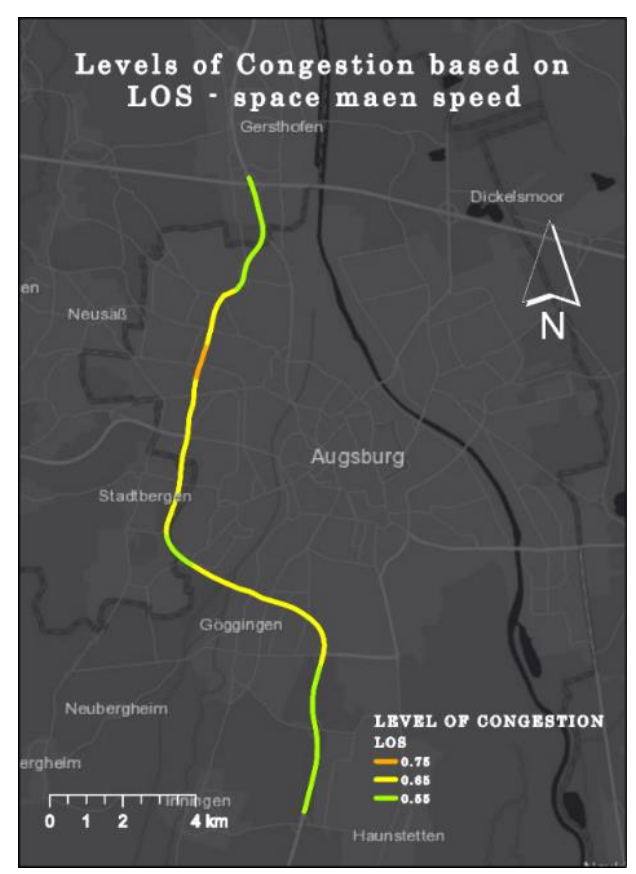

b) 


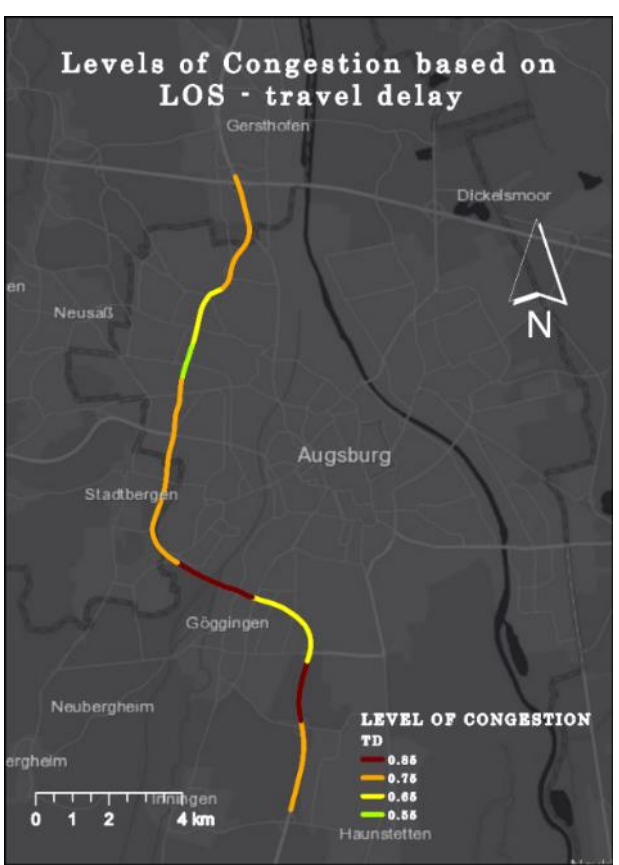

c)

Figure 3: Levels of congestion detected a) by fuzzy models with two input parameters - space mean speed and travel time \& b) based on Level of Service (LOS) space mean speed \& c) based on LOS travel delay(s). Color scheme goes from light green (no congestion), yellow (low congestion), orange (mild congestion), dark red (severe congestion) to black (extreme congestion).

\section{Conclusion and Outlook}

Traffic congestion is related with many factors, and usually shows difference at different location or in different time period and similarity or recurrence at similar conditions (Xu et al. 2013). Congested road segments are spatially close and temporally approximate. Initial findings indicate that a fuzzy inference model with two input variables (segment mean speed and travel time) is capable of identifying traffic congestions on both smaller and larger scale (segment and total road lengthwise).

Why do we not use a simple classification of our traffic parameters and make an analysis of the traffic conditions? In contrast to conventional methods (e.g. Level of Service - LOS based on space mean speed or on travel delay), fuzzy models deliver less sensitive results. This is possible due to capability of models to handle vague and imprecise data, as well as subjective judgment of real life conditions, all of which is integrated in the models. LOS based on space mean speed also provides satisfactory results, which confirms the theory that space mean speed is statistically stable and as such particularly applicable for short roadway segments or small travel times (Wardrop, 1952). LOS based on travel delay shows that this chunk of road is rather under mild to severe congestion most of the time. However, travel delay is a congestion measure defined as a ratio between actual and acceptable travel time (Lomax et al., 1997). This straightforward mathematical approach cannot handle high variations in input parameters, and as such often delivers error driven and noise sensitive outputs. Therefore, we would not recommend any further analysis using travel delays calculated based on standard Highway Capacity Manual (Handbuch für die Bemessung und Straßenverkehrsanlagen HBS, 2015), or at least not in combination with FCD solely.

Obtained results from the fuzzy model with segment mean speed and travel time parameters shows efficient performance in describing traffic behaviour at the investigated road segments. It is rather an approximation approach, yet capable of handling both noisy traffic data and road conditions (e.g. change in speed limit, duration of travel delay, etc.). The case study presents promising indication that fuzzy logic modelling approaches may give more reasonable results in contrast to commonly used analytical techniques because it takes more than one congestion measure and combines them into a composite image of traffic congestion. In the future work, we want to investigate this further by applying fuzzy inference model with segment mean speed calculated on a 10-minute interval to derive information about traffic on a finer scale. Additionally, the model(s) can be enriched with more input parameters, such as density or vehicle flow, to observe if the detected levels change and if so how significantly.

\section{References}

Adu-Gyamfi, Y., Sharma, A., Knickerbocker, S., Hawkins, N., and Jackson, M. (2015) Reliability of Probe Speed Data for Detecting Congestion Trends. IEEE 18th International Conference on Intelligent Transportation Systems, September 15-18, 2015. Las Palmas, Spain, https://doi.org/10.1109/ITSC.2015.362.

Aftabuzzaman, M. (2007) Measuring traffic congestionA critical review. In Proceedings of the 30th Australian Transport Research Forum (ATRF), Melbourne, Australia, 25-27 September 2007.

Boarnet, M.G., Kim, E.J. and Parkany, E. (1998) Measuring traffic congestion, Transportation Research Record: Journal of the Transportation Research Board, No. 1634, pp.93-99.

Bundesministerium für Verkehr und digitale Infrastruktur: Handbuch für die Bemessung und Straßenverkehrsanlagen (HBS), Bonn, den 26. August, $2015 . \quad$ https://www.verkehrsplanungweimar.de/PDF/HBS-Qualitaetsstufen-2015.pdf.

Davarynejad, M. and Vrancken, A. (2009) A survey of fuzzy set theory in intelligent transportation: State of the art and future trend. IEEE - International Conference on Systems, Man and Cybernetics, San Antonio, TX. 39523958. https://doi.org/10.1109/ICSMC.2009.5346648.

Fabritiis, C., Ragona, R., and Valenti, G. (2008) Traffic estimation and prediction based on real time floating car data. Proceeding of the 11th International conference on Intelligent Transportation Systems, October 12-15, 2008. Beijing, China, https://doi.org/ 10.1109/ITSC.2008.4732534. 
Fullér, R. and Zimmermann, H. (1993) Fuzzy reasoning for solving fuzzy mathematical programming problems. Fuzzy Sets and Systems, 60(3), 121-133.

Hamad, K. and Kikuchi, S. (2002) Developing a measure of traffic time congestion: fuzzy inference approach, Transportation Research Record: Journal of the Transportation Research Board, No. 1802, pp. 77-85.

He, F., Yan, X., Liu, Y., Ma, L. (2016) A traffic congestion assessment method for urban road networks based on speed performance index. Procedia Eng. 2016, 137, 425433.

Kalinic, M and Krisp, J.M. (2019) Fuzzy inference approach in traffic congestion detection. Annals of GIS, 25(3),329-336,

https://doi.org/10.1080/19475683.2019.1675760.

Kong, X., Xu, Z., Shen, G., Wang, J., Yang, Q., and Zhang, B. (2016) Urban traffic congestion estimation and prediction based on floating car trajectory data. Future Generation Computer System, 61, 97-107, https://doi.org/10.1016/j.future.2015.11.013.

Kosko, B., and Mitaim, S. (1996) What is the best shape for a fuzzy set in function approximation? Proceedings of the 5th IEEE International Conference on Fuzzy Systems, September 11 1996, New Orleans, USA, https://doi.org/10.1109/FUZZY.1996. 552354.

Li, Q., Ge, Q., Miao, L., and Qi, M. (2012) Measuring Variability of Arterial Road Traffic Condition Using Archived Probe Data. Journal of Transportation Systems Engineering and Information Technology, 12(2), 41-46, https://doi.org/10.1016/S1570-6672(11)60193-1.

Lee C.C. (1990) Fuzzy Logic in Control Systems: Fuzzy Logic Controller-Part I and part H. In: IEEE Transactions on Systems Man and Cybernetics 20(2):404 - 435.

Levinson, H.S. and Lomax, T.J. (1996) Developing a travel time congestion index, Transportation Research Record: Journal of the Transportation Research Board, No. 1564, pp. 1-10.

Lobo, A., Amorim, M., Rodrigues, C., and Couto, A. (2018) Modelling the Operating Speed in Segments of Two-Lane Highways from Probe Vehicle Data: A Stochastic Frontier Approach. Journal of Advanced Transportation, https://doi.org/10. 1155/2018/3540785.

Lomax T et al. (1997) Quantifying congestion. NCHRP Report. Washington, DC: Transportation Research Board,

http://onlinepubs.trb.org/onlinepubs/nchrp/nchrp_rpt_39 8.

Mamdani, E.H., and Assilian, S. (1975) An experiment in linguistic synthesis with a fuzzy logic controller. International Journal of Man-Machine Studies, 7(1), 113.

Mendel, J. (1995) Fuzzy logic systems for engineering. A tutorial. Proceedings of the IEEE 83, 83(3), 345-377.

Röss, R.P., Messer, C.J., Mschane, W.R., Fruin, J.J., Levinson, H.S., May, A.D. and Dudek, C.L. (1985)
Highway Capacity Manual. Special Report 209, Washington, D.C.: Transportation Research Board.

Sunderrajan, A., Viswanathan, V., Cai, W., and Knoll, A. (2016) Traffic State Estimation Using Floating Car Data. Procedia Computer Science, 80, 2008-2018, https://doi.org/10.1016/j.procs.2016.05.521.

Sugeno, M. (1985) An Introductory Survey of Fuzzy Control. In: Information Sciences, 36, 59-83.

Ter Huurne, D., Andersen, J. (2014) A Quantitative Measure of Congestion in Stellenbosch Using Probe Data. In Proceedings of the first International Conference on the use of Mobile Information and Communication Technology (ICT) in Africa UMICTA 2014, Stellenbosch, South Africa, 9-10 December 2014; ISBN 978-0-7972-1533-7.

Todorovic, D. (1994) Fuzzy sets theory applications in traffic and transportation. European Journal of Operational Research, 74(3). 389-390. https://doi.org/10.1016/0377-2217(94)90218-6.

Turner, S.M., Lomax, T.J. and Levinson, H.S. (1996) Measuring and estimating congestion using travel timebased procedures, Transportation Research Record: Journal of the Transportation Research Board, No. 1564, pp. 11-19.

Wardrop, J.G. (1952) Some Theoretical Aspects of Road Traffic Research. In Proceedings Institute of Civil Engineers, Road Engineering Division Meeting, January 1952, London, Great Britain.

Wan, C., Yang, Z., Zhang, D., Yan, X., Fan, S. (2018) Resilience in transportation systems: A systematic review and future directions. Transp. Rev. 2018, 38, 479498.

Wu, N. (2015) New Features in the 2015 German Highway Capacity Manual (HBS2015). Proceedings of the $14^{\text {th }}$ World Conference on Transport Research, 10-15 July 2016,

Shanghai, https://www.researchgate.net/publication/329774871_G erman_Highway_Capacity_Manual_HBS2015.

Zhao, N., Yu, L., Zhao, H., Guo, J., and Wen, H. (2009) Analysis of Traffic Flow Characteristics on Ring Road Expressways in Beijing: Using Floating Car Data and Remote Traffic Microwave Sensor Data. Transportation Research Record, 2124(1), 178-185, https://doi.org/10.3141/2124-17.

Xu, L., Yue, Y., and Li, Q. (2013) Identifying Urban Traffic Congestion Pattern from Historical Floating Car Data. Procedia - Social and Behavioral Sciences, 96, 2084-2095. https://doi.org/10.1016/j.sbspro.2013.08.235.

Zadeh, L.A. (1965) Fuzzy Sets. Information and Control, $8,338-353$.

Zadeh, L. (1973) Outline of a new approach to the analysis of complex systems and decision processes. IEEE Transactions on Systems, Man and Cybernetics SMC, 3, $28-44$. 
Zadeh, L.A. (1998) Knowledge representation in fuzzy logic. IEEE Transactions on Knowledge and data
Engineering,
$1(1)$,
89-100.

https://doi.org/10.1142/9789814261302_0039. 